\title{
Erratum to: Time-Periodic Solutions of the Navier-Stokes Equations in Unbounded Cylindrical Domains - Leray's Problem for Periodic Flows
}

\author{
H. BeirÃo dA VeIGA
}

Communicated by V. ŠVERÁK

\section{Erratum to: Arch. Rational Mech. Anal. 178 (2005) 301-325 DOI 10.1007/s00205-005-0376-3}

Equation (32) in the proof of Theorem 3 is incorrect. A correction can be done as follows. In going from equation (30) to equation (32), replace the test function $\phi$ by $w_{l}, l=1, \ldots, m$, (as opposed to $\left.\lambda_{l} w_{l}\right)$. Further, in equation (32), replace (only on the left-hand side) $k$ by $k \lambda_{l}^{-1}$. Finally, in the definition of the matrix $\mathcal{M}$ given in the original paper, namely,

$$
\mathcal{M}=\left[\begin{array}{ll}
M & k I \\
-k I & M
\end{array}\right]
$$

replace the matrices $k I$ by the diagonal matrix $K=k \operatorname{diag}\left[\lambda_{1}^{-1}, \ldots, \lambda_{m}^{-1}\right]$. The proof then follows as in the original paper. The statement of the theorem remains unchanged.

We use this occasion to correct a few misprints. In equation (20), page 308, the last symbol $\chi$ should be replaced by $A \chi$. On page 309 the real function $\alpha$ should satisfy not only $\alpha(a)=0$ but also $\alpha(b)=1$. In the second term on the right hand side of equation (27), $(b-a)$ should be replaced by $(b-a)^{2}$ and $u(t)$ by $u^{\prime}(t)$. Finally, in page 310 , replace $\gamma_{i j}=\delta_{i j}-\left(w_{j}, e\right)\left(e, w_{l}\right)$ by $\gamma_{j l}=\delta_{j l}-$ $\left(w_{j}, e\right)\left(e, w_{l}\right)$.

The author regrets the inconvenience.

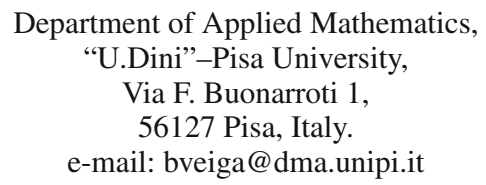

